\title{
Complexity Control of Fast Motion Estimation in H.264/MPEG-4 AVC with Rate- Distortion-Complexity optimization
}

\author{
Wu, Mo; Forchhammer, Søren; Aghito, Shankar Manuel
}

Published in:

Visual Communications and Image Processing 2007

Publication date:

2007

Document Version

Early version, also known as pre-print

Link back to DTU Orbit

Citation (APA):

Wu, M., Forchhammer, S., \& Aghito, S. M. (2007). Complexity Control of Fast Motion Estimation in H.264/MPEG-4 AVC with Rate-Distortion-Complexity optimization. In Visual Communications and Image Processing 2007: Proc. of SPIE-IS\&T Electronic Imaging (Vol. SPIE Vol. 6508, 650824, pp. 1-11). IS\&T and SPIE.

- Users may download and print one copy of any publication from the public portal for the purpose of private study or research.

- You may not further distribute the material or use it for any profit-making activity or commercial gain

- You may freely distribute the URL identifying the publication in the public portal 


\title{
Complexity Control of Fast Motion Estimation in H.264/MPEG-4 AVC with Rate-Distortion-Complexity optimization
}

\author{
$\mathrm{Mo} \mathrm{Wu}^{\dagger}$, Søren Forchhammer and Shankar M. Aghito \\ Department of Communications, Optics and Materials, COM•DTU, 2800 Lyngby, Denmark
}

\begin{abstract}
A complexity control algorithm for H.264 advanced video coding is proposed. The algorithm can control the complexity of integer inter motion estimation for a given target complexity. The Rate-Distortion-Complexity performance is improved by a complexity prediction model, simple analysis of the past statistics and a control scheme. The algorithm also works well for scene change condition. Test results for coding interlaced video (720 x576 PAL) are reported.
\end{abstract}

Keywords: H.264/AVC, Fast motion estimation, complexity control and inter motion estimation

\section{INTRODUCTION}

The recent H.264/MPEG-4 part 10 advanced video coding (AVC) standard $^{1,2}$ has improved encoding performance roughly by a factor of 2 compared to previous MPEG standards. ${ }^{5}$ The gain in coding efficiency comes at the price of a significant increase in encoding complexity, mainly due to new features in the inter motion estimation stage, which supports variable block sizes and multiple reference frames. Fast motion estimation may be applied to reduce complexity. To control the computational load and make optimal use of the available processing power, a control mechanism is desirable for real-time applications. To achieve this a computational complexity control is designed aiming at maintaining the rate-distortion (R-D) performance as much as possible with the given computational power. The new control method is based on rate-distortion-complexity (R-D-C) optimization.

The Enhanced Predictive Zonal Search (EPZS) fast motion estimation algorithm ${ }^{3}$ for integer inter motion estimation was implemented with some modifications for the H.264 reference software encoder in a previous work. ${ }^{4}$ Lagrangian optimization was applied to the extended EPZS algorithm, which is considered as a good fast motion estimation solution for the H.264/AVC codec. The video coding performance is traditionally measured by R-D performance. When complexity is an important factor, the performance may be measured in terms of R-D-C. An operational method for analyzing the optimal selection of the macroblock (MB) partition mode and number of reference frames in the motion compensation was developed. ${ }^{4}$ The basic idea is to transform the threedimensional problem of concurrently optimizing, e.g. minimizing R-D-C into a more tractable two-dimensional problem. The minor changes in distortion due to the complexity control are converted into small changes in rate by using a local slope of the R-D curve for the chosen parameter setting. This effectively eliminates the distortion parameter, and the optimization problem has thus been reduced to a problem of two parameters, modified rate $\left(R^{*}\right)$ and complexity $(C)$. For complexity control, a solution based on increasing or decreasing the percentage of skipped macroblocks using a Lagrangian R-D-C cost function has been proposed. ${ }^{6}$ In the following, Section 2 presents the control scheme in detail and Section 3 presents the experimental results.

\footnotetext{
${ }^{\dagger}$ Further author information: (Send correspondence to $\mathrm{Mo} \mathrm{Wu}$ )

Mo Wu: E-mail: mw@com.dtu.dk, Telephone: +45 45253620
} 


\section{MOTION ESTIMATION COMPLEXITY CONTROL}

A complexity control algorithm is designed for and integrated in the H.264/AVC reference software with an extended EPZS fast motion estimation ${ }^{4}$ method. The algorithm is designed for standard definition TV (SDTV) interlaced video with GOP structure, IBBPBBPBBPBB $\left(\operatorname{IBBP}_{(12)}\right)$. Inside each GOP, there are three BBP structures, hereafter denoted as P-GOPs. The scheme is applied at each P-GOP, by controlling the parameters of the encoder's configuration. In this algorithm, the parameters of the configuration that can be adaptively changed are the partition modes and the reference fields that can be allowed for inter motion estimation. ${ }^{2}$ The generalization to other GOP structures is straightforward. Only the integer inter motion estimation complexity is considered.

A limitation on computational resources leads to a bound on the complexity, $C$. Under this constraint, the R-D performance shall be optimized. Transforming R-D into $R^{*}{ }^{4}$ the optimization is based on considering the slope, $\frac{\delta R^{*}}{\delta C}$, where $\delta R^{*}$ is the small change of the $R^{*}$ due to variation of the complexity, $\delta C$. Thus when complexity needs to be decreased as part of the control process, it can be done by reducing the search space, and skipping the settings that produce the lowest $\left|\frac{\delta R^{*}}{\delta C}\right|$.

\subsection{Calculating the integer inter motion estimation complexity}

Before describing the control scheme, notation and the method for calculating the integer inter motion estimation complexity is presented in Sections 2.1.1 and 2.1.2. The same notation is used in the following sections.

\subsubsection{Weighted search positions}

The inter motion estimation complexity is measured by a weighted number of search positions, which is a simple measurement of the motion search complexity. ${ }^{4}$ The number of weighted search positions of different block partitions are counted according to the block size in inter mode motion estimation (see Table 1).

Table 1. Weighted search positions of different block partitions per search

\begin{tabular}{|c||c|c|c|c|c|c|c|}
\hline Block partition & $16 \times 16$ & $16 \times 8$ & $8 \times 16$ & $8 \times 8$ & $8 \times 4$ & $4 \times 8$ & $4 \times 4$ \\
\hline Weighted search positions per search & 16 & 8 & 8 & 4 & 2 & 2 & 1 \\
\hline
\end{tabular}

The parameter indicating the partition modes for frame $i$ is $b(i)$, which is defined by

$$
b(i)= \begin{cases}1, & \text { block partition } 16 \times 16 \\ 2, & \text { block partition down to } 8 \times 8 \\ b_{\max }=3, & \text { all block partitions }\end{cases}
$$

The method is designed for interlaced video, so one frame includes two fields. The parameter indicating the reference fields for frame $i$ is $r(i)$. For $r(i)=1$, if the $i_{t h}$ frame is a $\mathrm{P}$ frame, the search is restricted to the first two reference fields in the reference list ${ }^{2}$ for forward prediction; if the $i_{t h}$ frame is a $\mathrm{B}$ frame, an additional frame is used for backward prediction. ${ }^{2}$ As $r(i)$ increases by one, an extra reference field for forward prediction can be searched. In the experiments, the maximum number of the reference frames is five, so the maximum value of $r(i), r_{\max }=9$.

Thus the weighted search positions in frame $i$ are a function of $b(i)$ and $r(i)$, and indicated as $C_{P F}(b(i), r(i), i)$ and $C_{B F}(b(i), r(i), i)$ for $\mathrm{P}$ and $\mathrm{B}$ frames, respectively, if the other parameters are left unchanged throughout the encoding process.

For a window of $M$ frames, we define the total complexity,

$$
C=\sum_{i \in \mathrm{P} \text { frames }}^{M} C_{P F}(b(i), r(i), i)+\sum_{i \in \mathrm{B} \text { frames }}^{M} C_{B F}(b(i), r(i), i),
$$


where

$$
\left\{\begin{array}{l}
C_{P F}(b(i), r(i), i)=\sum_{b=1}^{b(i)} \sum_{r=1}^{r(i)} C_{P}(b, r, i) \\
C_{B F}(b(i), r(i), i)=\sum_{b=1}^{b(i)} \sum_{r=1}^{r(i)} C_{B}(b, r, i)
\end{array}\right.
$$

where $C_{P}(b, r, i)$ and $C_{B}(b, r, i)$ are the number of weighted search positions searching on the $r_{t h}$ reference fields and $b_{t h}$ partition modes (shown in Eq. (4)) in the $i_{t h}$ frame for a $\mathrm{P}$ and $\mathrm{B}$ frame, respectively. In the sum in Eq. (2), the notation $\mathrm{i} \in \mathrm{P}$ frames is not strictly correct, since I frames, where one field is encoded with inter motion estimation, should also be included in the sum.

$i \in \mathrm{P}$ frame is a general restriction, it may include the I frame $i$, if frame $i$ has one field, which is also encoded with inter motion estimation.

$$
b= \begin{cases}1, & \text { partition }: 16 \times 16 \\ 2, & \text { partitions }: 16 \times 8,8 \times 16 \text { and } 8 \times 8 \\ 3, & \text { partitions }: 8 \times 4,4 \times 8 \text { and } 4 \times 4\end{cases}
$$

\subsubsection{Weighted number of coded blocks}

Later we shall evaluate the effect of decreasing the number of motion searches. This could be based on the occurrence of actually chosen combinations so far. $N_{P}(b, r, i)$ and $N_{B}(b, r, i)$ are the weighted number of coded blocks that are encoded by referring to the $r_{t h}$ reference fields and with the partition mode selected from the $b_{t h}$ partition modes for frame $i$, which is $\mathrm{P}$ and $\mathrm{B}$ frame, respectively. Because of SKIP mode, direct mode, bi-prediction etc of H.264/AVC codec, the counting method of $N_{P}(b, r, i)$ and $N_{B}(b, r, i)$ are not as simple as that of $C_{P}(b, r, i)$ and $C_{B}(b, r, i)$. In a $\mathrm{P}$ frame's skip mode or a B frame's direct mode, there is no coded motion vectors. The encoder and the decoder will use the same predicted motion vectors (MVs), constructed from previously compressed MVs. Thus, except for the compression of MVs, the same motion compensation scheme is applied. Thus it is reasonable to count them into $N_{P}(b, r, i)$ or $N_{B}(b, r, i)$. For collecting the statistics, the counters go through all the macroblocks, and for each macroblock, the counting method is shown in Figure 1.

\subsection{Complexity prediction model}

In the control scheme, a given target weighted search positions per second, $C_{\mathrm{T}}$, is provided for a window of $M$ frames. The controller may increase or decrease the number of search positions according to $C_{\mathrm{T}}$. Thus, a motion search complexity prediction model is required to predict the complexity that will be used for coding the following frames with different configurations, and select the appropriate parameters. The model below is applied for adjusting the settings when a (small) increase in complexity is possible and desired.

Through the analysis of the statistics of three training sequences, Mobcal, Cycling and Barcelona, an elaborate method is applied to predict the complexity of the next P-GOP for a given configuration. According to terms

used in the previous sections, the complexity of the P-GOP starting at the $i_{t h}$ frame, e.g. the index of the first B frame is $i$, is defined as

$$
C_{B B P}(b(i), r(i), b(i+2), r(i+2), i)=\sum_{j=i}^{i+1} C_{B F}(b(j), r(j), j)+C_{P F}(b(i+2), r(i+2), i+2),
$$

where $b(i+1)=b(i)$ and $r(i+1)=r(i)$, i.e. the two B frames have the same settings, $b(i)$ and $r(i)$, while $b(i+2)$ and $r(i+2)$ indicate the settings for the $\mathrm{P}$ frame. In the following, for a shorter notation, $b(i), r(i), b(i+2)$ and $r(i+2)$ are replaced by $b_{i}^{\prime}, r_{i}^{\prime}, b_{i}$ and $r_{i}$, respectively. When only the $16 \times 16$ partition mode and one reference frame are used for both $\mathrm{P}$ and $\mathrm{B}$ frames in a P-GOP, the complexity can be described as,

$$
C_{B B P}(1,1,1,1, i)=C_{P F}(1,1, i+2)+\sum_{j=i}^{i+1} C_{B F}(1,1, j) .
$$




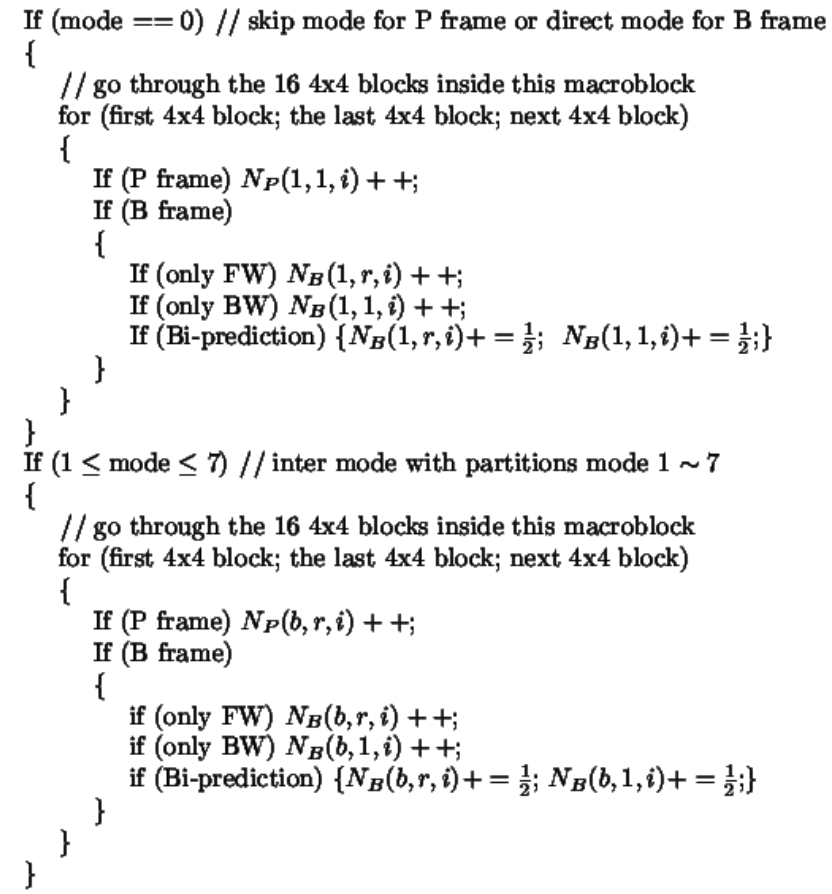

Figure 1. The method of updating the counters, $N_{P}(b, r, i)$ and $N_{B}(b, r, i)$ over one macroblock for the frame $i$. FW refers to forward prediction, BW refers to the backward prediction, and the mode is the same notation as defined in H.264 standard. ${ }^{1}$

Let $\hat{C}(\cdot)$ denotes the prediction of $C(\cdot)$. Let $C_{B B P}\left(b_{n}, r_{n}, b_{n}^{\prime}, r_{n}^{\prime}, n\right)$ indicate the complexity of the next PGOP; based on the previous collected statistics, $C_{B B P}(1,1,1,1, i), C_{B B P}\left(b_{n}, r_{n}, b_{n}^{\prime}, r_{n}^{\prime}, n\right)$ is obtained as follows,

$$
\begin{aligned}
\hat{C}_{B B P}\left(b_{n}, r_{n}, b_{n}^{\prime}, r_{n}^{\prime}, n\right) & =\hat{C}_{P F}\left(b_{n}, r_{n}, n+2\right)+\sum_{j=n}^{n+1} \hat{C}_{B F}\left(b_{n}^{\prime}, r_{n}^{\prime}, j\right) \\
& =\left(f_{P 1}\left(b_{n}, r_{n}, n\right)+f_{B 2}\left(b_{n}^{\prime}, r_{n}^{\prime}, n\right)+1\right) \cdot C_{B B P}(1,1,1,1, i),
\end{aligned}
$$

where

$$
f_{P 1}\left(b_{n}, r_{n}, n\right)=\frac{\hat{C}_{P F}\left(b_{n}, r_{n}, n+2\right)-C_{P F}(1,1, i+2)}{C_{B B P}(1,1,1,1, i)}= \begin{cases}k_{0}\left(r_{n}-1\right), & \text { if } b_{n}=1 \\ k_{1}\left(r_{n}-1\right)+k_{2}, & \text { if } b_{n}=2 \\ k_{3}\left(r_{n}-1\right)+k_{4}, & \text { if } b_{n}=3\end{cases}
$$

and

$$
f_{B 2}\left(b_{n}^{\prime}, r_{n}^{\prime}, n\right)=\frac{\sum_{j=n}^{n+1} \hat{C}_{B F}\left(b_{n}^{\prime}, r_{n}^{\prime}, j\right)-\sum_{j=n}^{n+1} C_{B F}(1,1, j)}{C_{B B P}(1,1,1,1, i)}= \begin{cases}k_{5}\left(r_{n}^{\prime}-1\right), & \text { if } b_{n}^{\prime}=1 \\ k_{6}\left(r_{n}^{\prime}-1\right)+k_{7}, & \text { if } b_{n}^{\prime}=2 \\ k_{8}\left(r_{n}^{\prime}-1\right)+k_{9}, & \text { if } b_{n}^{\prime}=3,\end{cases}
$$

where $k_{0}=0.168, k_{1}=0.56, k_{2}=0.6, k_{3}=0.82, k_{4}=1.05, k_{5}=0.4, k_{6}=1.34, k_{7}=2.0, k_{8}=2.0$ and $k_{9}=3.6$, and the mathematic model reflects the linear relation between the search complexity used for $\mathrm{P}$ and $\mathrm{B}$ frames and $r_{n}$ and $r_{n}^{\prime}$, respectively. Because the model is designed for complexity prediction based on fast motion estimation method, the linear relation between the complexity used and the applied different block partitions does not simply exist. The parameters, $k_{m}$, are experimentally determined by collecting the results of 


$$
\frac{\sum_{i} C_{P F}\left(b_{i}, r_{i}, i\right)+\sum_{i} C_{B F}\left(b_{i}^{\prime}, r_{i}^{\prime}, i\right)}{\sum_{i} C_{B B P}(1,1,1,1, i)},
$$

for the three test sequences, and then calculate the average value, which is assumed to be expressed by

$$
\frac{E\left[C_{P F}\left(b_{i}, r_{i}, i\right)\right]+2 \cdot E\left[C_{B F}\left(b_{i}^{\prime}, r_{i}^{\prime}, i\right)\right]}{E\left[C_{B B P}(1,1,1,1, i)\right]}
$$

where $E[\cdot]$ denotes expectation. The statistics are fitted to the model determining parameters, $k_{m}$.

Similarly, derived from Eq. (7), $\hat{C}_{B B P}\left(b_{n}, r_{n}, b_{n}^{\prime}, r_{n}^{\prime}, n\right)$ can also be predicted based on a given previous P-GOP complexity, $C_{B B P}\left(b_{i}, r_{i}, b_{i}^{\prime}, r_{i}^{\prime}, i\right)$

$$
\hat{C}_{B B P}\left(b_{n}, r_{n}, b_{n}^{\prime}, r_{n}^{\prime}, n\right)=\frac{\left(f_{P 1}\left(b_{n}, r_{n}, n\right)+f_{B 2}\left(b_{n}^{\prime}, r_{n}^{\prime}, n\right)+1\right)}{\left(f_{P 1}\left(b_{i}, r_{i}, i\right)+f_{B 2}\left(b_{i}^{\prime}, r_{i}^{\prime}, i\right)+1\right)} \cdot C_{B B P}\left(b_{i}, r_{i}, b_{i}^{\prime}, r_{i}^{\prime}, i\right),
$$

\subsection{Measurement of benefit terms}

When decreasing the complexity, we evaluate the marginal performance of $b$ and $r$ for $\mathrm{P}$ frame and $b^{\prime}$ and $r^{\prime}$ (the same definition as $b$ and $r$, respectively) for B frames, e.g. $b=2$ compared with $r^{\prime}=3$. The aim is to determine values of $b$ or $r$ or $b^{\prime}$ or $r^{\prime}$ that do not (currently) efficiently utilize (in terms of $R^{*}$ ) the available computational complexity $C$. These are found by evaluating, before encoding the current P-GOP, the benefit terms, $\Lambda(\cdot)$, defined as

$$
\begin{array}{cc}
\Lambda_{P}(b, i)= & s_{P} \cdot B_{b}(i+2) \cdot \frac{\sum_{r_{s}} N_{P}\left(b, r_{s}, i+2\right)}{\sum_{r_{s}} C_{P}\left(b, r_{s}, i+2\right)}, \\
\Lambda_{P}(r, i) & =s_{P} \cdot B_{b}(i+2) \cdot \frac{\sum_{b_{s}} N_{P}\left(b_{s}, r, i+2\right)}{\sum_{b_{s}} C_{P}\left(b_{s}, r, i+2\right)}, \\
\Lambda_{B}\left(b^{\prime}, i\right) & =\frac{1}{2} \sum_{j=i}^{i+1}\left(B_{b}(j) \cdot \frac{\sum_{r_{s}} N_{B}\left(b^{\prime}, r_{s}, j\right)}{\sum_{r_{s}} C_{B}\left(b^{\prime}, r_{s}, j\right)}\right), \\
\Lambda_{B}\left(r^{\prime}, i\right) & =\frac{1}{2} \sum_{j=i}^{i+1}\left(B_{b}(j) \cdot \frac{\sum_{b_{s}} N_{B}\left(b_{s}, r^{\prime}, j\right)}{\sum_{b_{s}} C_{B}\left(b_{s}, r^{\prime}, j\right)}\right),
\end{array}
$$

where $\Lambda_{P}(b, i)$ and $\Lambda_{B}\left(b^{\prime}, i\right)$ are the benefits selecting $b_{t h}$ and $b_{t h}^{\prime}$ partition modes for the $\mathrm{P}$ and $\mathrm{B}$ frames, respectively, in the previous P-GOP, while $\Lambda_{P}(r, i)$ and $\Lambda_{B}\left(r^{\prime}, i\right)$ are the benefits selecting $r_{t h}$ and $r_{t h}^{\prime}$ reference fields for the $\mathrm{P}$ and $\mathrm{B}$ frames, respectively. $B_{b}(i)$ is the number of bits utilized for coding the frame $i$. The scaling factor $s_{P}=2$ is introduced in order to prioritize $\mathrm{P}$ frames, which are more important, since they may be used as reference frames for B frames. The maximum number of reference frames/fields is set to $r_{\max }=9$. The ratio, $\frac{\sum_{r_{s}} N_{P}\left(b, r_{s}, i\right)}{\sum_{r_{s}} C_{P}\left(b, r_{s}, i\right)}$ as an example, could be viewed as the weighted number of blocks per complexity unit that can be improved using the partition modes of $b$ for coding the $\mathrm{P}$ picture. The computational load due to motion estimation may be decreased by skipping the search for those combinations of picture type and configuration that correspond to the smallest benefit terms, $\operatorname{argmin}\left\{\Lambda_{P}(b, i), \Lambda_{P}(r, i), \Lambda_{B}\left(b^{\prime}, i\right), \Lambda_{B}\left(r^{\prime}, i\right)\right\}$. This model has been constructed based on experiments.

\subsection{Control scheme}

For each sequence, best individual settings ${ }^{4}$ may be determined in a R-D-C sense. If the sequence has 'stationary' properties over the whole sequence, it can be assumed that each segment of the sequence also has the same best individual settings and R-D-C performance. Because the $\mathrm{R}-\mathrm{D}$ performance has been simplified as $\mathrm{R}^{*}$, the $\mathrm{R}^{*}-\mathrm{C}$ curve decided by best individual settings is denoted by $\mathrm{R}_{\mathrm{opt}}^{*}-\mathrm{C}$. By construction, $\mathrm{R}_{\mathrm{opt}}^{*}$ is a convex function of $C$. A simple mathematical model of each segment is obtained as follow: the $\mathrm{R}^{*}$ value of the $k_{t h}$ segment is 
denoted as $\Delta \mathrm{R}_{\mathrm{opt}}^{*(k)}$ and it is calculated using the best individual settings. $\Delta \mathrm{R}_{\mathrm{opt}}^{*(k)}$ can be viewed as a function of the complexity used in segment $k$, and the complexity is denoted by $\Delta \mathrm{C}^{(k)}$. i.e., $\Delta \mathrm{R}_{\text {opt }}^{*(k)}=\mathrm{g}\left(\Delta \mathrm{C}^{(k)}\right)$.

Let us assume 'stationarity', which we shall use for steady state operation (State 0 below), and the sequence is divided in $\mathrm{N}$ segments of equal length of frames. If the same setting is utilized in every segment, thus $\Delta C^{(k)}=\Delta C^{(l)}, \forall 1 \leq k, l \leq N, k \neq l$, regardless of the start of the sequence. So,

$$
\begin{aligned}
\Delta C^{(k)} & =\Delta C=\frac{C}{N}, \\
\Delta R_{o p t}^{*(k)} & =\Delta R_{o p t}^{*}=\frac{R_{o p t}^{*}}{N} .
\end{aligned}
$$

When the complexity control is applied, the best settings used can be different for each segment of the sequence. In this case the complexity measure for the $k_{t h}$ segment, $\Delta C^{\prime}(k)$, is not constant across the whole sequence. With the complexity control scheme,

$$
N \cdot \Delta C=\sum_{i}^{N} \Delta C^{\prime(k)}
$$

Jensen's inequality states that if $g(x)$ is a convex function, $E[g(X)] \geq g(E[X]) .{ }^{8}$ So,

$$
R_{o p t}^{*}=N \cdot \Delta R_{o p t}^{*}=N \cdot g(\Delta C)=N \cdot g\left(E\left[\Delta C^{\prime(k)}\right]\right) \leq N \cdot E\left[g\left(\Delta C^{\prime(k)}\right)\right]=\sum_{k=1}^{N} g\left(\Delta C^{\prime(k)}\right)=\sum_{k=1}^{N} \Delta R_{o p t}^{*^{\prime}(k)}=R_{o p t}^{*},
$$

where $R_{o p t}^{*}$ is the $R^{*}$ obtained by selecting the same best individual settings for each segment, while $\Delta R_{o p t}^{*^{\prime}(k)}$ is the $\mathrm{R}^{*}$ obtained in the $k_{t h}$ segment by the control scheme, i.e. when different settings are allowed for different segments. Eq. (20) shows: a better $\mathrm{R}^{*}-\mathrm{C}(\mathrm{R}-\mathrm{D}-\mathrm{C})$ performance compared with the best fixed setting is not achievable, if the best individual settings of the R-D-C are the exact same over the short uniform segments of a 'stationary' video sequence. So, the control scheme not only needs to control the complexity according to the target complexity, but also should avoid dramatically changing the complexity in each P-GOP.

A control scheme (given below) is designed in order to efficiently make use of the available computational power, maintain the R-D $\left(R^{*}\right)$ performance and avoid dramatic changes in complexity. It includes scene change detection and control of the fast motion estimation at scene change. The details of the steady state control process is shown below as the main part of the control scheme in State 0. State $\mathbf{1}$ - $\mathbf{3}$ handles transitions at the beginning and after a scene change.

The processing of states 0 - 3 are described in pseudo code below.

State 0: This is the steady state control state. Before encoding one P-GOP, the scene change detector will check if a scene change is taking place, if yes, go to state 1 , otherwise stay in state 0 .

if (scene change detected) go to State 1

else

if (all reference frames are present) // in the first few P-GOPs, the reference frames specified

by the parameters of the configuration may not be present, e.g. the first P-GOP cannot search on the $5_{t h}$ reference frame \{

if $\left(C_{\mathrm{P}-\mathrm{GOP}}>C_{\mathrm{P}-\mathrm{GOPT}}^{(i)}\right) / / C_{\mathrm{P}-\mathrm{GOP}}$ is the complexity used in the previous P-GOP. $C_{\mathrm{P}-\mathrm{GOPT}}^{(i)}$ refers to Eq. (23)

\{

while $\left(C_{\mathrm{P}-\mathrm{GOP}}^{\prime}+C_{\mathrm{P}-\mathrm{GOP}}>2 \cdot C_{\mathrm{P}-\mathrm{GOPT}}^{(i)}\right) / / C_{\mathrm{P}-\mathrm{GOP}}^{\prime}$ is the predicted complexity if a subset in the setting is switched off 


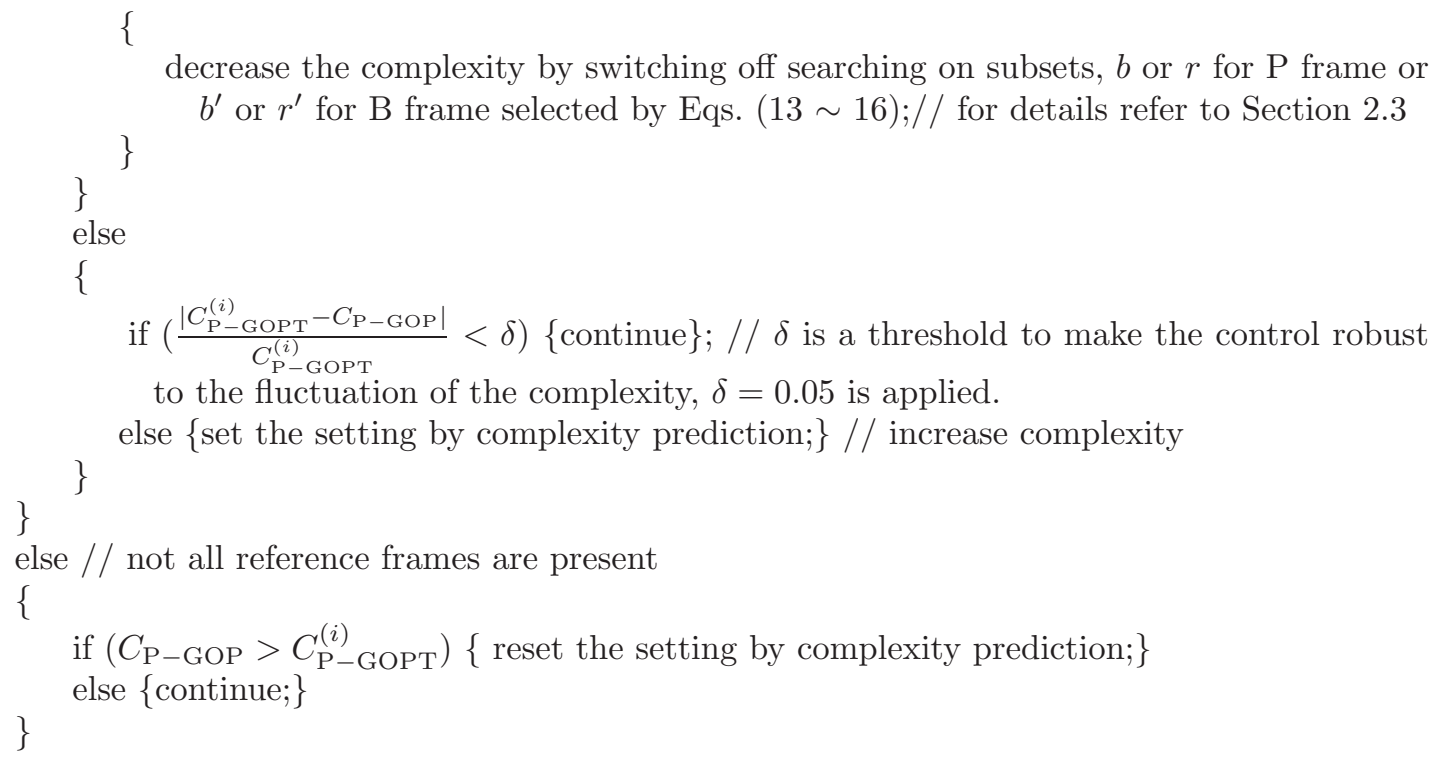

State 1: After a scene change is detected, the configuration with the $16 \times 16$ partition mode and one reference frame is selected for the next $\mathrm{P}$ frame. Searching down to $4 \times 4$ partition mode and one reference frame is selected for B frames. Finish encoding the current P-GOP, then Go to State 2.

State 2: Configuration with searching down to $4 \times 4$ partition mode and one reference frame is selected for both $\mathrm{P}$ and $\mathrm{B}$ frames. Finish encoding the current P-GOP, then Go to State 3.

State 3: Configuration with searching down to $4 \times 4$ partition mode and one reference frame plus one reference field is selected for both $\mathrm{P}$ and $\mathrm{B}$ frames. Finish encoding the current P-GOP, switch between the block partition priority or reference priority preset orders by comparing $\frac{\sum_{b_{s}=1}^{b} N_{P}\left(b_{s}, 2, i\right)}{\sum_{b_{s}=1}^{b} N_{P}\left(b_{s}, 1, i\right)}$ with a predefined threshold, $T$; set configuration by complexity prediction model; Go to State 0 .

In the control process, there is a target complexity, $C_{T}$. The complexity control method can adaptively change the target complexity of P-GOP, $C_{\mathrm{P}-\mathrm{GOPT}}$, which has linear relation with $C_{T}$. The relation between $C_{T}$ and average target complexity of P-GOP, $\overline{C_{\mathrm{P}-\mathrm{GOPT}}}$, is mainly affected by the frame rate and GOP structure. As an approximation, it is assumed that there is no complexity for I frames and complexities of $\mathrm{P}$ and $\mathrm{B}$ frames are viewed as the same. Thereafter, the relation can be calculated by

$$
\begin{gathered}
C_{T}=\eta \cdot \overline{C_{\mathrm{P}-\mathrm{GOPT}}}, \\
\eta=\frac{(p-1) \cdot f}{p \cdot p^{\prime}},
\end{gathered}
$$

where $p$ is the period of one GOP, $p^{\prime}$ is the period of one P-GOP and $f$ is the frame rate. In conducting the experiments, the GOP structure is $I B B P B B P B B P B B_{(12)}$, thus $p=12, p^{\prime}=3$ and $f=25$ (frames/s), so $C_{T}=7.6389 \cdot \overline{C_{\mathrm{P}-\mathrm{GOPT}}}$. In practice, the relation is also slightly affected by $C_{T}$ and the limited number of settings that can be chosen. Thus $C_{\mathrm{P}-\mathrm{GOPT}}$ should be adaptively controlled according to $C_{T}$ for the test sequences. To achieve this, the target P-GOP complexity after coding the most recent frame, $i_{t h}$ frame, is

$$
C_{\mathrm{P}-\mathrm{GOPT}}^{(i)}=\frac{C_{T} \cdot M-f \cdot \sum_{j=1}^{j=i}\left[C_{P F}(b(j), r(j), j)+C_{B F}(b(j), r(j), j)\right]}{\eta \cdot(M-i)} .
$$




\section{EXPERIMENTAL RESULTS}

The adaptive complexity control method was applied to PAL $(720 \times 576$ pixels, 25 fps $)$ test sequences. The GOP structure is $\operatorname{IBBP}_{(12)}$. R-D optimization is enabled in the reference software. Direct mode is tested in spatial mode.

The commonly used test sequences Mobcal, Barcelona and Table tennis are utilized (each with 100 frames). The accuracy of the complexity control is shown in Table 2. On the average the proposed scheme controls the integer motion estimation complexity with an accuracy of $0.43 \%$ of the target complexity. The complexity is measured in millions of weighted search positions per second (MWSP/s). ${ }^{4}$

The coding performance for Mobcal, Table tennis and Barcelona are shown in Figure 2, where the $\mathrm{R}^{*}$-C curves obtained with the proposed complexity control are compared to those obtained in previous work ${ }^{4}$ by using the best average settings, the best individual settings and the complexity worst case settings (for details refer to Appendix A). best average settings and best individual settings are all obtained using off-line optimization of the parameters, and they are acquired by coding the test sequences with all the settings. The complexity worst case settings obtained represents a conservative design approach based on the analysis of the complexity worst case. We note that the adaptive scheme controlling complexity on-line outperforms the off-line optimization of fixed settings. The main improvements are seen for the low target complexities, which is our main objective. (At the higher complexity levels, the methods basically converge to searching the full configuration $b_{\max }$ and $r_{\max }$ ) For target complexities, $C_{\mathrm{T}}<210 \mathrm{MWSP} / \mathrm{s}$, the three test sequences' average improvement of the $R^{*}$ compared with best individual settings is $0.76 \%$; compared with best average settings is $1.70 \%$ and improvements up to $7.48 \%$ are obtained for the sequence Mobcal and low complexities; compared with the complexity worst case settings, it is $6.3 \%$ in average and improvements up to $18 \%$.

Table 2. The measured complexity per second, $C_{s}$, achieved by the proposed control scheme compared to the target complexity $C_{\mathrm{T}}$. Complexities are measured in MWSP/s. The range is roughly $50-450 \mathrm{MWSP} / \mathrm{s}$, depending on the sequence.

\begin{tabular}{|c||ccc|ccc|}
\hline Sequence & Barcelona & Mobcal & Tab.ten. & MobBar & MobTab & BarTab \\
\hline mean $\left(\left|C_{\mathrm{T}}-C_{s}\right|\right)$ & 0.94 & 0.76 & 0.84 & 0.78 & 0.36 & 0.51 \\
mean $\left(\frac{\left|C_{\mathrm{T}}-C_{s}\right|}{C_{s}}\right)$ & $0.42 \%$ & $0.44 \%$ & $0.44 \%$ & $0.32 \%$ & $0.17 \%$ & $0.19 \%$ \\
\hline
\end{tabular}

Scene changes occur frequently in video. Three test sequences with a scene change are constructed, each by concatenating two of the sequences above. The new sequences are named MobBar (Mobcal + Barcelona), MobTab (Mobcal + Table) and BarTab (Barcelona + Table). The $\mathrm{R}^{*}-\mathrm{C}$ results for MobBar and BarTab are shown in Figure 3. When $C_{\mathrm{T}}<210 \mathrm{MWSP} / \mathrm{s}$, the three test sequences' average improvement of $R^{*}$ compared with best individual settings is $2.38 \%$, compared with best average settings, it is $2.62 \%$, compared with the complexity worst case settings, it is $7.19 \%$. The average complexity control accuracy is $0.2 \%$ (shown in Table 2 ).

\section{CONCLUSIONS}

A new adaptive complexity control algorithm is presented. Besides accurately controlling the computational load of motion estimation during the encoding process aimed at real-time implementations, it also provides slight average improvements in R-D performance compared to the previously found off-line optimized settings. ${ }^{4}$ These improvements are considerable in some cases, up to $6.3 \%$ compared with the off-line solution. The improvements is up to $18 \%$ compared with the complexity worst case settings.

\section{APPENDIX A. RESULTS TO BE COMPARED WITH}

The following measures are used for comparison. Let $r_{M}$ and $b_{M}$ refer to a fixed setting of $r(i)$ and $b(i)$ for all $i$ in the window of $M$ frames.

The best individual settings are found among all the individually-tested settings for each sequence based on convex optimization of $\mathrm{R}^{*}-\mathrm{C}$. 
The best average settings $\left(S_{\text {avg }}\right)$ are found among all tested settings that the three initial test sequences (training datasets) have in common and averaging both the $R^{*}$ and the $C$ values. They are denoted by $R_{\text {avg }}^{*}$ and $C_{\text {avg }}$. The best average settings are decided by convex optimization. The set based on these sorted (by complexity) settings is denoted as $S_{\text {avg }}$.

The complexity worst cases settings are decided by the following process. First, find the worst-case Complexity by testing the individual settings $\left(r_{M}, b_{M}\right)^{4}$ for sequences, giving 15 different combinations of $\left(r_{M}, b_{M}\right)$ for each, out of these find the largest complexity of different sequences for each setting as the worst-case complexity.

$$
C_{\text {worst case }}\left(r_{M}, b_{M}\right)=\max \left(C_{\text {train dataset } 1}\left(r_{M}, b_{M}\right), C_{\text {train dataset } 2}\left(r_{M}, b_{M}\right) \ldots\right)
$$

Second, for a given $\mathrm{C}_{\mathrm{T}}$, calculate all possible settings with smaller complexity,

$$
S_{1}=\left\{\left(r_{M}, b_{M}\right) \mid C_{\text {worst case }}\left(r_{M}, b_{M}\right) \leq C_{T}, \forall\left(r_{M}, b_{M}\right)\right\}
$$

Finally, the $R^{*}$ of the complexity worst cases settings are calculated by

$$
R^{*}\left\{\operatorname{argmin}\left[R_{\text {avg }}^{*}\left(r_{M}, b_{M}\right)\right]\right\},\left(r_{M}, b_{M}\right) \in S_{1} \bigcap S_{a v g}
$$

\section{REFERENCES}

1. T. Wiegand and G. J. Sullivan (ed.), "Draft ITU-T Rec. and FDIS (ITU-T Rec. H.264-ISO/IEC 14496-10 AVC)," H.264/MPEG-4 AVC (JVT-doc. JVT-G050), Mar. 2003.

2. T. Wiegand et al., "Overview of the H.264/AVC video coding standard," IEEE Trans. Circuits and Systems for Video Tech., vol. 13, no. 7, pp. 1-19, July 2003.

3. A. M. Tourapis, "Enhanced predictive zonal search for single and multiple frame motion estimation," in Proc. SPIE VCIP 2002, San Jose, CA, USA, 2002, pp. 1069-1079.

4. J. S. Andersen, S. Forchhammer and S. M. Aghito, "Rate-Distortion-Complexity optimization of Fast Motion Estimation in H.264/MPEG-4 AVC," in Proc. ICIP 2004, Singapore, Oct. 2004, pp. 111-114.

5. D. Alfonso et al., "Detailed rate-distortion analysis of H.264 video coding standard and comparison to MPEG2/4," in Proc. SPIE VCIP 2003, Lugano, Switzerland, 2003, pp. 891-902.

6. C. Kannangara, and I. Richardson, "Computional Control of An H.264 Encoder Through Lagrangian Cost Function Estimation", in Proc. VLBV'05, Sardinia, Italy, Sept. 2005, p. 4.

7. I. Richardson, H.264 and MPEG-4 Video Compression, John Wiley \& Sons Ltd, 2003.

8. K. Sayood. Introduction to Data Compression. Morgan Kaufmann, second edition, 2000. 


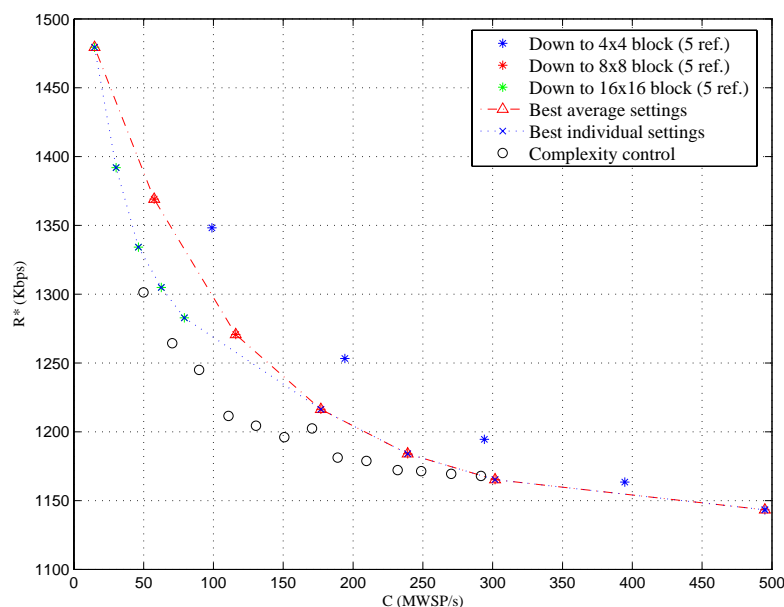

(a)

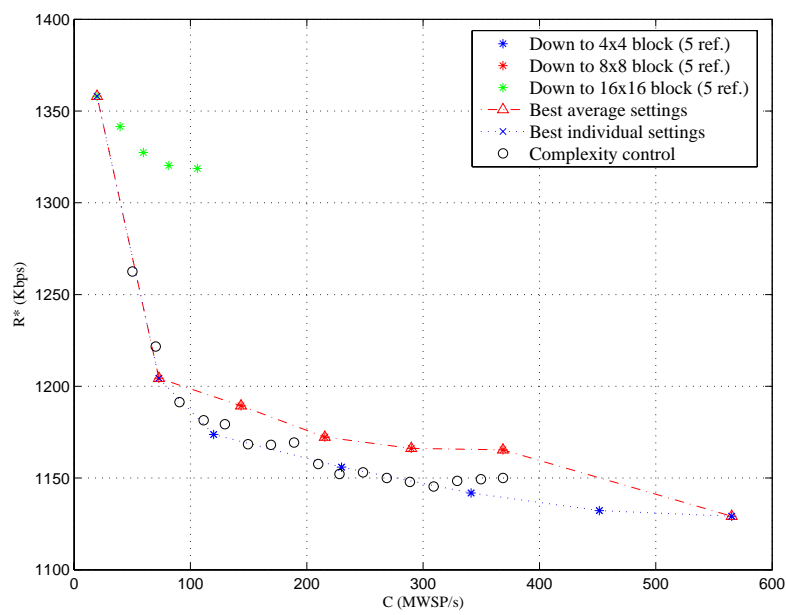

(c)

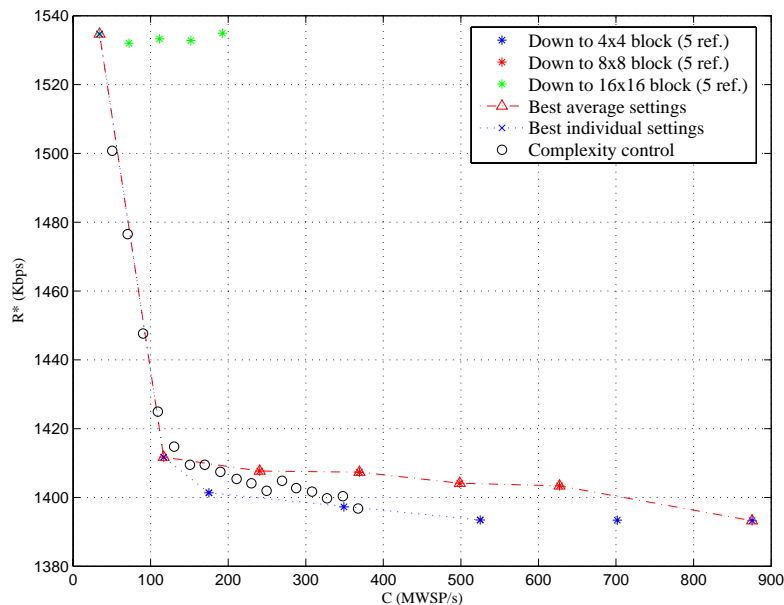

(e)

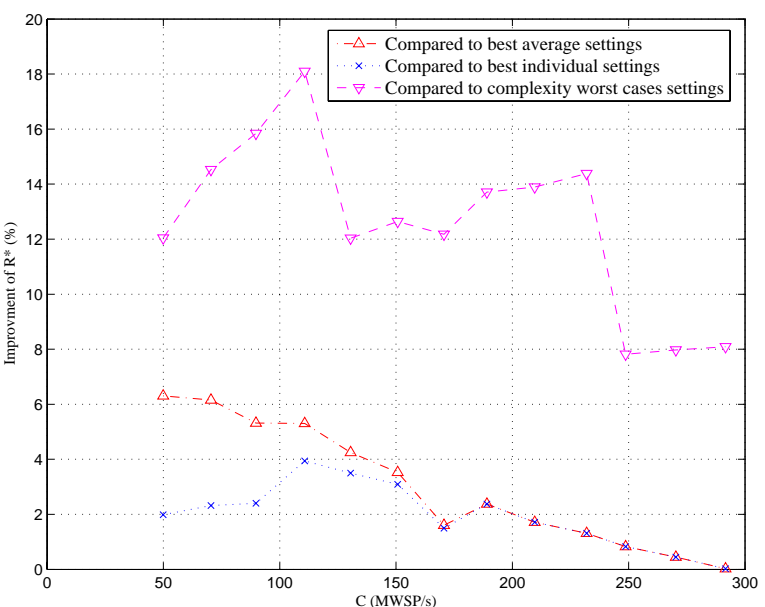

(b)

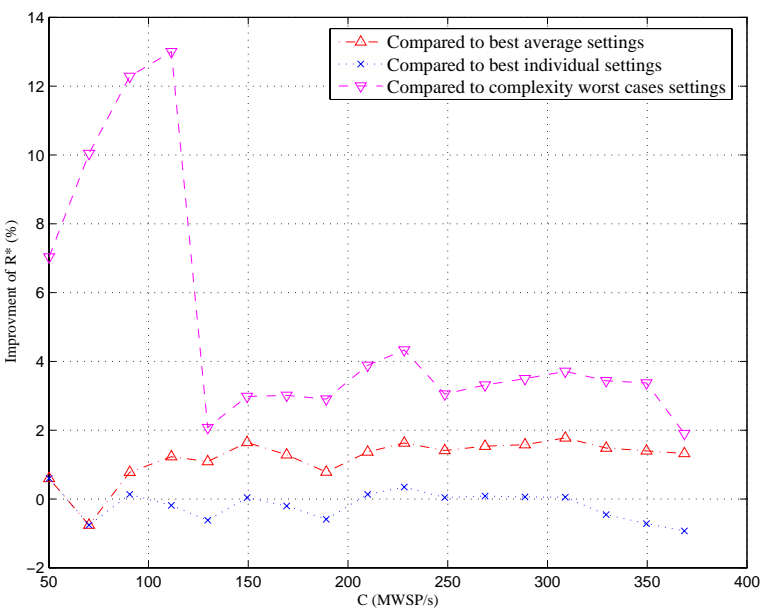

(d)

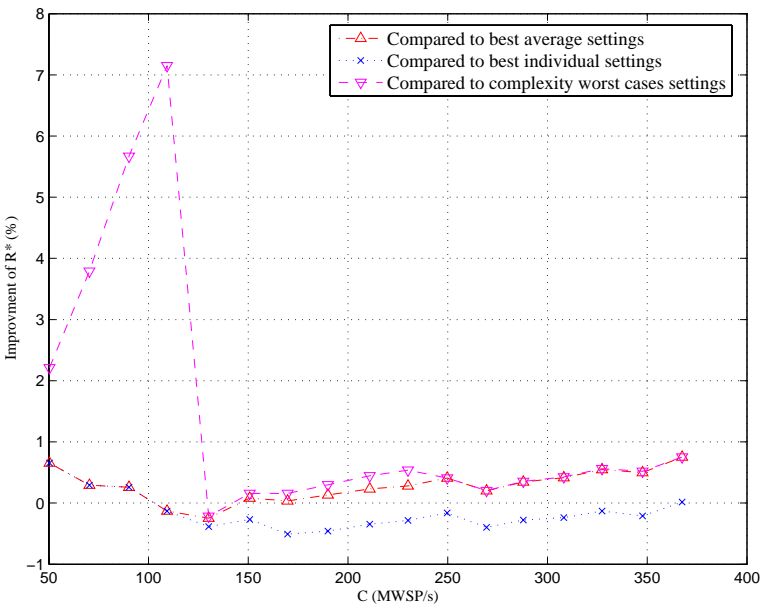

(f)

Figure 2. Evaluation of the R-D-C $\left(\mathrm{R}^{*}-\mathrm{C}\right)$ performance: The proposed control scheme compared to the best average settings, the best individual settings and the complexity worst case settings, for Mobcal (a), Table tennis (c) and Barcelona (e). The percentage of improvement in terms of $R^{*}$, for Mobcal (b), Table tennis (d) and Barcelona (f). 


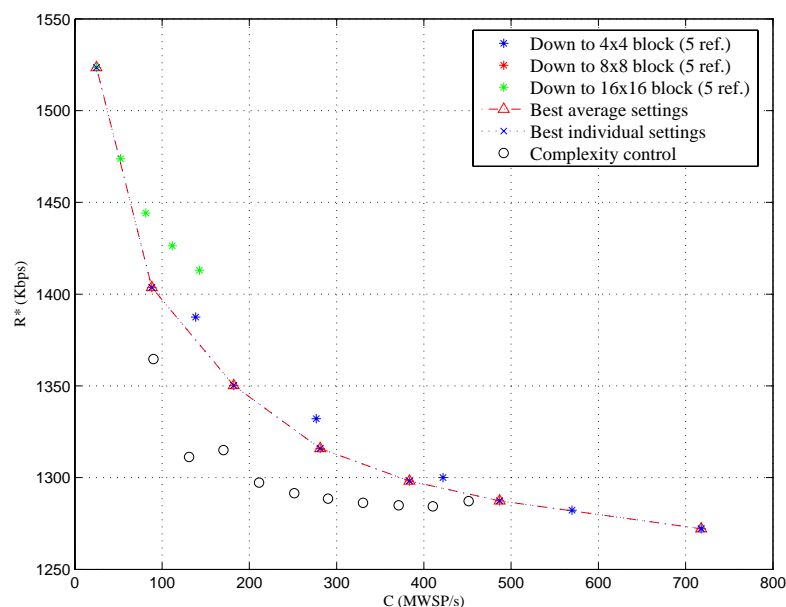

(a)

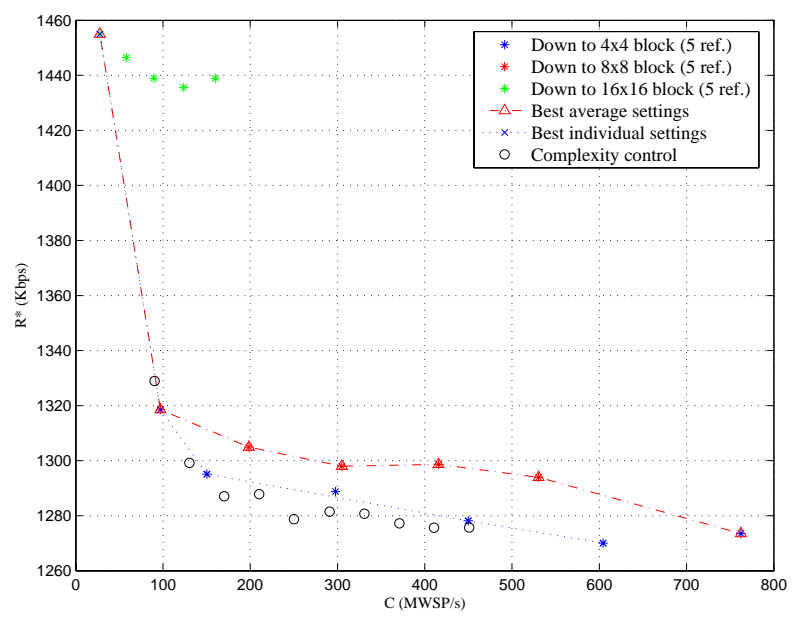

(c)

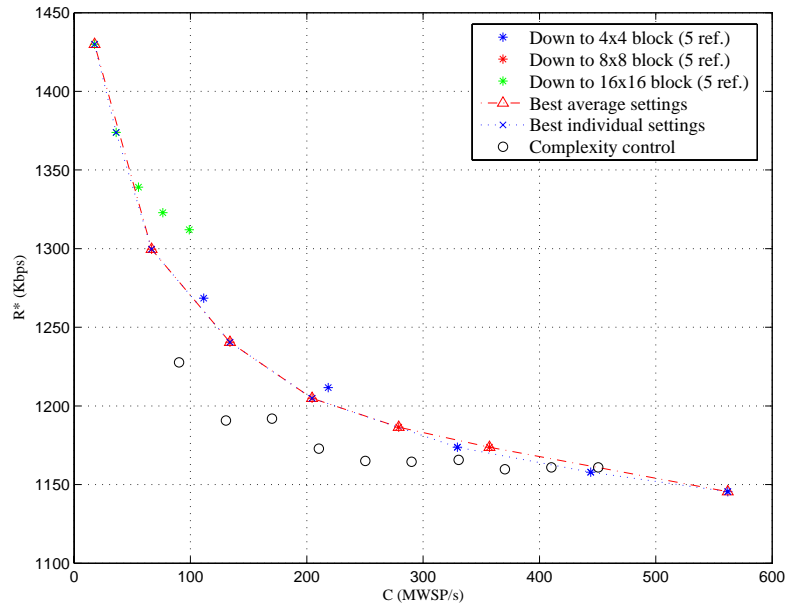

(e)

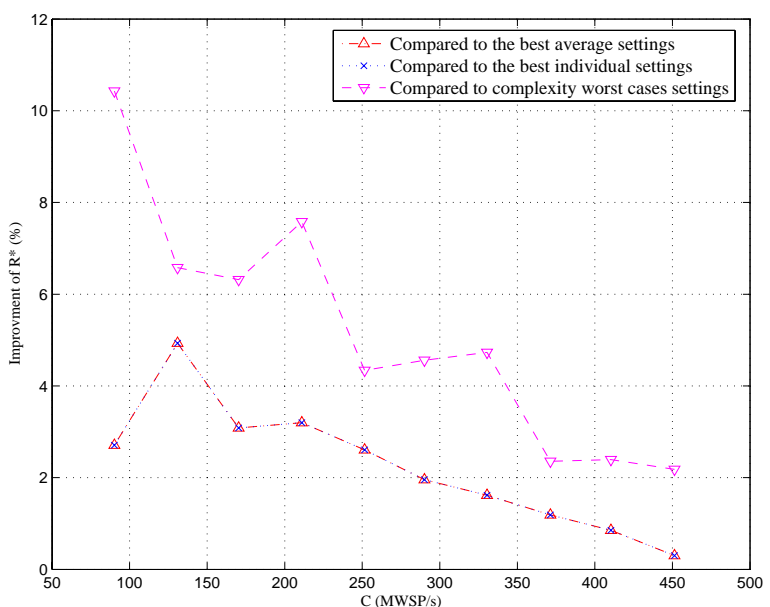

(b)

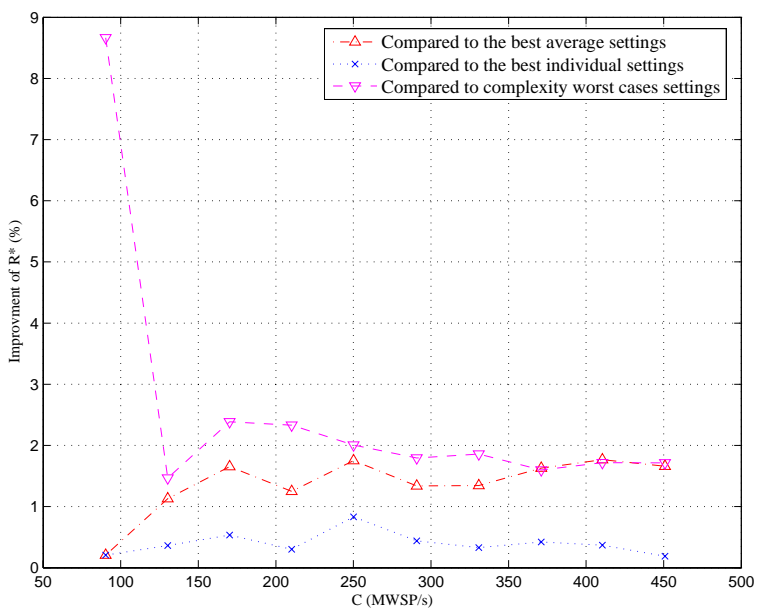

(d)

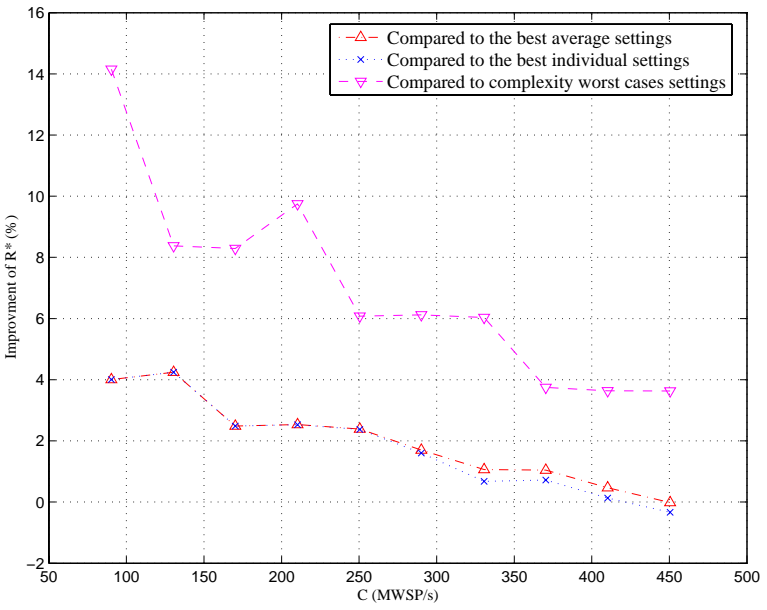

(f)

Figure 3. Evaluation of R-D-C $\left(\mathrm{R}^{*}-\mathrm{C}\right)$ performance for sequences with scene change. The proposed scheme compared to the best average settings, the best individual settings and the complexity worst case settings, for MobBar (a), BarTab (c) and MobTab (e). The percentage of improvement in terms of $R^{*}$, for MobBar (b), BarTab (d) and MobTab (f). 